Voix et Images

voixetimages

\title{
Le poème retourné contre soi
}

DENISE BRASSARD

Volume 42, numéro 3 (126), printemps-été 2017

URI : https://id.erudit.org/iderudit/1041054ar

DOI : https://doi.org/10.7202/1041054ar

Aller au sommaire du numéro

Éditeur(s)

Université du Québec à Montréal

ISSN

0318-9201 (imprimé)

1705-933X (numérique)

Découvrir la revue

Citer ce compte rendu

BRASSARD, D. (2017). Compte rendu de [Le poème retourné contre soi]. Voix et Images, 42(3), 137-145. https://doi.org/10.7202/1041054ar d'utilisation que vous pouvez consulter en ligne.

https://apropos.erudit.org/fr/usagers/politique-dutilisation/ 


\author{
P O ÉS I E \\ Le poème retourné contre soi \\ $+++$
}

DENISE BRASSARD

Université du Québec à Montréal

Dès les premières pages de La main hantée ${ }^{1}$, j'ai été happée par la tonalité si terriblement juste, puis j'ai ressenti le désir de lire à voix haute, de dire à mon tour cette parole, de la partager. Car ici, me semble-t-il, plus encore qu'ailleurs dans la poésie de Louise Dupré, où elle est pourtant omniprésente, l'adresse interpelle le lecteur, directement, au plus intime de la souffrance.

Le livre s'ouvre sur un aveu de fragilité, et la fragilité en est la véritable signature. Une femme conduit chez le vétérinaire son chat malade pour le faire euthanasier, voilà le motif de départ. Cet événement en apparence anodin, par les questions qu'il pose, prend peu à peu un pouvoir de résonance et des proportions planétaires. Le chat hurlait, hurlait. La femme, n'en pouvant plus de l'entendre, s'est laissé entraîner vers la fatalité. La cruauté de cet acte commis de façon machinale - de quel droit en a-t-elle décidé ainsi? - la hante jour et nuit:

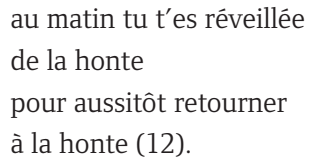

Cette honte, qu'elle lit dans les yeux de son chat, dit la violence qui la visite en rêve, mais qui n'en est pas moins réelle: celle à laquelle nous assistons au quotidien, impuissants, souvent indifférents. Dans une volonté de porter un ultime secours à l'animal, la voix berce, et on se laisse bercer, comme le chat qui s'abandonne aux étapes du "protocole». Cherchant à (se) consoler, à (se) rassurer, la femme se projette dans une mort semblable, paisible, trouvée au terme d'une simple injection. Mais sa bonne conscience est sans cesse troublée par la culpabilité qui la ronge. Après tout, elle fait disparaître son chat sans son consentement, «comme une fille sa mère» (25). Aussi le bercement s'avère-t-il trompeur (ou trompé?) et même insidieux, car sans cesse sujet aux arrêts brusques ou aux emballements, si bien qu'en réalité la voix vous traverse comme une flèche, vous tétanise, vous laissant perclus de douleur: «tu

1 Louise Dupré, La main hantée, Montréal, Éditions du Noroît, 2016, 113 p. 
lui dis des mots/muets de mère//tu lui touches la joue/en songeant aux condamnés/ achevés dans la haine» (20).

Écrire signifie ici dire ce qui ne se dit pas. Or pour pouvoir le dire, et surtout le faire entendre, il faut creuser «dans la langue/commune//une même fosse/aux bêtes et aux femmes» (23), creuser jusqu'à «toucher/la moelle de la langue» (27) et débusquer le côté humain, trop humain de la violence:

\author{
tu appartiens à une race \\ domestiquée \\ à l'ombre des églises \\ mais tu parles encore \\ comme tout le monde \\ la langue \\ de la barbarie (28-29);
}

Tu as sur les mains l'odeur millénaire du feu et du sang. (35)

Dire ce qui ne se dit pas implique également de consentir à descendre au fond de soi, afin d'explorer les coins les plus sombres de sa conscience, et de là remonter l'histoire humaine jusqu'à retrouver «l'humilité de [s]on corps d'insecte» (36).

«Tu as commencé à écrire, la main hantée. Tu n'habites pas seule ta souffrance et tu le sais.» (109) Bêtes maltraitées à mort, sorcières brûlées avec leurs chats, Indiennes violées et pendues aux arbres, écrivaines et écrivains ayant succombé au désespoir, tous elle les convoque, les rassemble, les prend sur ses genoux, les porte à son sein. Cette multitude pose la question de l'identité du sujet, voire de la subjectivité elle-même (l'agent de la mort est-il un sujet transcendant? souverain? libre?). Mais voilà: "Comment écrire je si on ne croit plus à l'espèce humaine?» (38) C'est là la question fondamentale que pose le livre, et peut-être bien celle qui est au centre de toute la poésie de Louise Dupré, où l'adresse autoréflexive est constante. Le je, outre la solidité qu'il exige, menace d'aveuglement (la tentation de l'ego), et s'il n'est pas accueil, il est refus. Il ne peut s'écrire que s'il se donne comme creuset de l'être pluriel ${ }^{2}$. Mais cela demeure à l'état de souhait, voire d'utopie, et le tu subsiste, qui semble mieux à même d'assumer l'ouverture et la prise en charge de la complexité, car en plus de postuler la scission du sujet, il met en rapport d'intimité le bourreau et la victime que chacun porte en soi. Ce n'est certainement pas par hasard que la porosité, la poussière, la suie sont aussi importantes dans ce recueil. Ainsi la femme se met dans la peau de son chat, cherche à vivre ce qu'il a vécu, décompose le réel jusqu'à la moindre parcelle afin de pouvoir

2 «Tu pourrais alors écrire je, comme si ce pronom se creusait enfin, devenait caverne, pierre poreuse qu'il suffirait de caresser de la paume pour que surgisse de l'oubli la forme des fossiles.» (39); "Car tu n'es pas sans fautes, tu commences à le reconnaître. Tu vois maintenant le jour où tu l'avoueras. Quand tu seras assez solide pour écrire je.» (42); «[T]u es un nom divisé par deux» (72). 
l'absorber. En consentant à monter elle-même sur le bûcher, elle se mêle à la poussière des siècles et, une fois devenue poreuse comme la pierre, se laisse pénétrer par la lumière. Car «la leçon de la lumière» (72) ne peut se répéter qu'à voix basse, comme le poème ne peut s'écrire que dans l'humilité, et c'est en fouillant dans la boue et dans la cendre qu'on retrouve des mots comme "charité», "amour», des mots que le jour traverse sans aveugler. Aussi n'est-ce pas sur un hypothétique retour au je que se clôt le recueil, mais sur un nous que la poète lance tel «un appel à témoin» (114).

Consentir ainsi à sa fragilité, à celle de l'écriture, avancer sans savoir où l'on va ni qui l'on est, c'est non seulement accueillir la multitude, c'est aussi permettre au poème de se retourner contre soi. Contre lui-même, d'abord, en tant qu'il est un langage susceptible d'autotélisme, alors qu'il s'agit de porter sur le réel un regard sans complaisance, mais aussi contre celle qui l'écrit, devant qui il se fait obstacle, objet de résistance sur quoi percutent la honte et l'absurdité de la souffrance. Dans ce retournement apparaît "l'ombre de la beauté», et alors celle qui avait toujours reconnu sa «dette envers la douleur, [...] soudain reconn[aît] sa dette envers la joie» (106). La joie, c'est petit, ça n'est ni éloquent ni éblouissant. C'est une lumière réfractée, diffractée par le corps devenu friable. Le revers du poème, sa seule promesse: ce qu'il dévoile une fois les images des souvenirs éclatées.

Manifestement au sommet de son art, Louise Dupré donne à lire une parole clairvoyante, toute en nuances, d'une lucidité et d'une sensibilité extrêmes. Avec une apparente économie de moyens, qui sert on ne peut mieux le projet du livre (simplicité, lexique familier, narrativité), tout en travaillant savamment le rythme (alternance et relance de la prose et du vers, coupures et enjambements, répétitions), elle joue admirablement de l'ombre et de la lumière. C'est ainsi que de trouée en trouée on traverse le deuil de cet amour perdu. C'est peu - le sujet ne se laisse berner ni par sa douleur ni par les prouesses du langage - et c'est beaucoup. Car l'écriture, affirme Louise Dupré, est notre seul rempart contre la barbarie, le seul lieu où l'on puisse résister au désespoir sans pour autant tomber dans le déni:

\author{
tu n'es pas dupe \\ de ton manège \\ mais tu écris \\ pour que ton sang \\ ne rougisse pas \\ le monde \\ tu l'as juré (65).
}


Jean-Simon DesRochers fait un retour en poésie avec Les espaces ${ }^{3}$, un recueil au caractère formaliste accusé, cérébral, souvent abstrait, mais bien maîtrisé, même si j'aurais souhaité que la dernière partie soit plus longue, laquelle donne à l'ensemble un sens rétrospectif, puisque tout en somme s'y délie, mais un peu trop rapidement, si bien qu'on retient assez peu de choses d'une première lecture. Mais peut-être était-ce là l'objectif de l'auteur, dont l'entreprise consiste précisément à retourner le passé comme un gant afin d'en épuiser les images.

Le poème initial est programmatique: les motifs qui structurent le recueil sont donnés dès la première strophe ${ }^{4}$. Ce sont autant de bornes sur le parcours d'une vie qu'on remonte de l'âge adulte jusqu'à l'enfance, soulevant au passage un amas d'images, lancinantes, indélogeables. Tout ce qu'on peut faire, c'est de les déplacer, pour en éprouver le sens, peut-être, ou encore la résistance, ou bien pour en conjurer la portée traumatique. On pourrait résumer le sujet du livre en citant cette belle phrase de Jérôme Meizoz: «La vie hurle et plante ses serres en vous, on se retourne pour voir d'où est venu le coup: appeler ça des souvenirs ${ }^{5}$.»

Ici, comme dans La main hantée, il est question d'identité problématique, incertaine, fragmentée, liée notamment à une conscience exacerbée du langage. L'identité du sujet est mise à l'épreuve de la perte, en même temps qu'elle se heurte à ce qui encombre sa mémoire et lui rend étranger son propre passé. De nouveau le retournement - du temps, des images, du poème contre soi - s'avère indispensable à l'actualisation de la subjectivité:

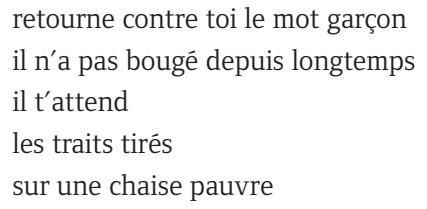

Les motifs se présentent comme des topoï, au sens rhétorique du terme, c'est-à-dire comme des lieux d'ancrage de la mémoire et (de clignotement) du sens; une fois activée, la mémoire travaille à déloger, à libérer les violences qui se sont cristallisées en eux. La méthode privilégiée est la projection et la prolifération des images jusqu'à ce qu'elles se décomposent en pixels puis disparaissent. D'où l'omniprésence des

3 Jean-Simon DesRochers, Les espaces, Montréal, Les Herbes rouges, 2016, 101 p. Depuis la parution de son dernier recueil (Parle seul, Montréal, Les Herbes rouges, 2003, 68 p.), il a publié principalement des romans.

4 «[Ā] travers toi se préciseront ces motifs :/une chaise/des familles écoles aux cités d'écrans/la piste menant à ton lit.» (9) «Motifs», «Chaise», «Familles», «Écoles», «Cités», «Écrans», «Piste» et «Lit» sont les titres des différentes parties qui composent le livre.

5 Jérôme Meizoz, Père et passe, Lausanne/Cognac, Éditions d'en bas/Le temps qu'il fait, 2008, p. 14. L'auteur souligne. 
miroirs, des écrans. En retour, le constant déplacement des images, leur remixage, semble rendre les miroirs et les écrans à un état liquide, voire même organique, ce qui les rend plus facilement apprivoisables.

Loin de miser sur la proximité et l'intimité, comme le fait Louise Dupré, JeanSimon DesRochers accuse la distance et l'accentue, mais cela produit un résultat semblable, soit une chute de l'être dans l'étant (ou du présupposé dans le mot) qui entraîne une extrême lucidité et commande l'humilité:

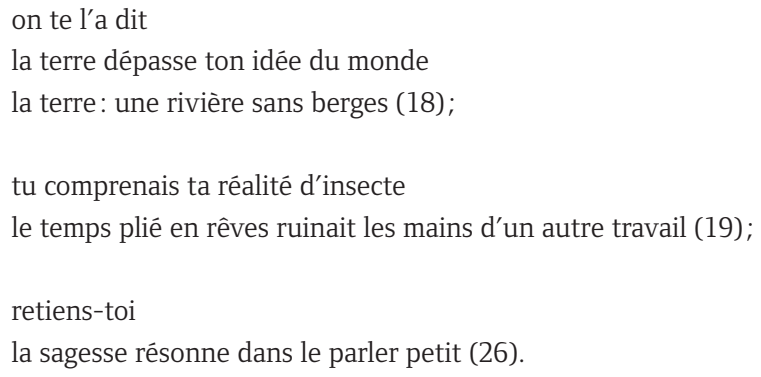

Cette humilité ne va toutefois pas sans un soupçon d'ironie. Car s'il dénote la violence des ordres que les parents et autres figures d'autorité balancent aux enfants sans se soucier des marques qu'ils sont susceptibles de laisser, ce «retiens-toi» connote aussi bien la violence intériorisée, celle que l'enfant, une fois devenu grand, se ressert à lui-même à la moindre occasion. Opérant dans la diachronie, la violence rejouée libère à la fois le garçon et l'adulte. Mais ce qui passe dans cette subtile ironie et dans le silence que figure l'entrechoquement de ces vers souvent opaques, c'est peutêtre aussi bien l'amour des parents et des maîtres, un amour entravé parce qu'ils ne surent pas le dire, parce qu'on ne leur avait pas appris:

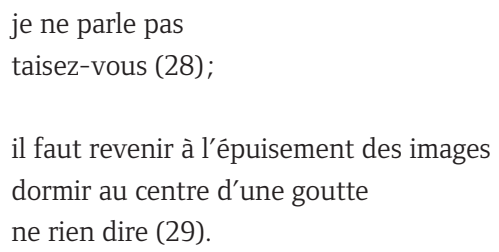

Cette tentative de corriger l'enfance prend la forme d'une rêverie un peu obsessionnelle: tout se déplace et se mélange, à commencer par les places que vous assignent les familles. C'est à cette seule condition que le sujet peut de nouveau prendre la mesure du monde, dont le rapport de familiarité qu'on croit avoir avec lui est en réalité un rapport d'étrangeté ${ }^{6}$ :

6 Ainsi que le rappelle Giorgio Agamben dans une relecture de Être et temps de Heidegger: «[C]'est à partir de cette neutralisation de l'être-à-portée-de-main que [...] peut être proposée la thèse singulière selon laquelle l'intimité avec le monde "est un mode de dépaysement [...] du Dasein et non l'inverse. Du point de vue ontologico-existential, le ne-pas-se-sentir-chez-soi [...] doit être conçu comme le phénomène le 
moi le garçon d'elle

divisé en garçon lui

je pose mes loques contre le sel famille

de ma sueur famille (34);

je nous veux binaires sans distance (36);

à peine vivant déjà miroir (56).

Être miroir, c'est ne pas avoir d'identité propre, c'est se laisser coloniser par celle d'autrui. Or l'image doit être projetée hors de soi - éprouver la résistance des objets, de ce qui lui fait obstacle - pour que l'identité s'actualise, et que le lien soit renoué entre le garçon et l'adulte. D'où l'importance des écrans, qui relayent en quelque sorte les miroirs et en complètent le mouvement.

Tant l'hermétisme que la composition ${ }^{7}$ fonctionnent ainsi suivant un principe de réfraction et de diffraction: les images, se renvoyant les unes aux autres, se gonflent, se multiplient, puis s'épuisent et finissent par disparaître au profit de l'espace qu'elles circonscrivent:
si petit dieu la tête
devient le meilleur écran de l'homme
j'y projetterai mes enfants hermétiques
ils rendront aux écoles leurs pardons
coffres à jouets et cortèges de semences
jetés à nos visages (55).

Porté à son paroxysme, cet effet spéculaire placé au cœur de l'entreprise ${ }^{8}$ se mue en «carnaval et son habit de vitre» (54), sorte de présence holographique, pas pleinement tangible, mais déjà plus qu'un rêve, et qui permet, paradoxalement, d'appréhender l'espace: un vide. Ainsi l'identité du sujet ouvre sur une sorte d'unité dialectique. Il s'agit moins d'accueillir la multitude, comme chez Louise Dupré, que d'investir la distance qui sépare le père du fils, l'homme de l'enfant, et d'en faire le lieu du déploiement de l'identité.

plus originaire".» Giorgio Agamben, Homo Sacer, vol. 4.2: L'usage des corps, traduit de l'italien par Joël Gayraud, Paris, Éditions du Seuil, coll. «L'ordre philosophique», 2015, p. 80.

7 Je pense ici en particulier à «Cités», dont les poèmes sont formés d'un premier texte en prose où des appels de notes renvoient à des extensions-développements. On dirait autant de petits univers - la révolution des gloses renvoyant au centre de chacun - à la fois en expansion et menacés d'implosion. Ainsi semblent se construire les cités. Dans la suite intitulée «Piste», où un poème en prose est suivi d'un poème en vers qui en reprend certains éléments, comme pour le condenser ou en extraire la substance, on retrouve un mouvement similaire, mais inversé.

8 Cela va jusque dans l'intégration de références nombreuses, jamais identifiées dans le texte, mais parfois facilement repérables, comme la très manifeste présence - ou influence? - de Roger DesRoches dans l'érotisme des poèmes de la suite «Écran». 
Dans la dernière suite, à laquelle tout le reste nécessairement menait et dont les poèmes, en général moins abstraits, sont très touchants, c'est clairement à luimême que le sujet s'adresse: "du calme:/reprends ton souffle et ta monnaie» (93). Ici, il n'est plus question d'ironie, car celui qui prend la mesure de l'espace, c'est bien lui, l'adulte, le sujet de l'énonciation, l'être sinon unifié, du moins recentré. Se confirme alors l'objectif de la quête apparu dès les premiers poèmes : vider le langage de l'illusion de l'être afin de libérer l'être des ombres du langage:

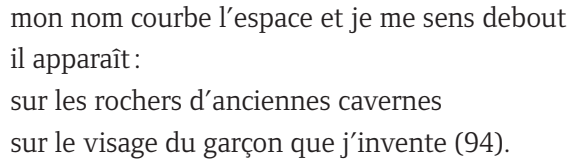

Il m'a semblé, on l'aura compris, que le souvenir de Platon hantait ce livre. Est-ce l'effet d'un simple jeu de l'auteur à partir de son nom, voire du hasard? Quoi qu'il en soit, cette piste vaudrait la peine d'être explorée. Car à cette entreprise de mémoire paraît associée une critique de l'ontologie, laquelle, en passant par Aristote (dont la scission de l'être ${ }^{9}$ est au fondement de la métaphysique), déboucherait sur une sorte de cratylisme spéculaire. Comme le rappelle Giorgio Agamben, en Occident, depuis Kant jusqu'à Heidegger, le sujet transcendantal est tenu pour acquis. Ce sont des philosophes tels Nietzsche, Benjamin, Foucault, mais aussi un linguiste comme Benveniste, qui ont fait basculer le sujet transcendantal vers le sujet parlant; «le locuteur s'est ainsi substitué au sujet transcendantal de Kant, la langue a pris la place de l'être comme a priori historique ${ }^{10}$ ». Tout en reconnaissant l'identité intrinsèquement liée à la langue, Jean-Simon DesRochers semble viser à en sortir le sujet: le garçon relégué à la fiction, l'homme se trouve libéré des ombres du passé et de la déchirure qu'elles perpétuent. Depuis les mots mêmes qui le définissent et le déterminent, le sujet, poussé aux limites de l'abstraction du langage, bascule dans l'espace mondain, où il se trouve tout entier livré à la contingence. Une fois les images dissoutes, le sujet, délesté du poids de l'être, devenu transparent, perméable à l'air, apparaît tel qu'en lui-même il se dit, ou tel qu'en réalité, peut-être, il est: «un espace comme les autres» (102).

Il est aussi question de familles et de filiation dans le premier recueil de Mélanie Landreville, un livre pénétrant qui porte le beau titre de Vertiges de l'hospitalitét ${ }^{11}$. Ici encore, l'identité pose problème, et ce sont principalement la honte et la peur qui en sont la cause. Ces sentiments qu'on se transmet de mère en fille, il importe d'en

9 «Dans le dispositif ontologique qu'Aristote lègue à la philosophie occidentale, la scission de l'être en essence et existence et l'introduction du temps dans l'être sont l'œuvre du langage. C'est la subjectivation de l'être comme hypokeimenon, comme ce-sur-quoi-il-est-dit, qui met en marche le dispositif.» Giorgio Agamben, Homo Sacer, vol. 4.2: L'usage des corps, p. 189.

10 Ibid., p. 170.

11 Mélanie Landreville, Vertiges de l'hospitalité, Montréal, Les Herbes rouges, 2016, 68 p. 
suivre la trace jusqu'à leur origine afin d'ébranler le verbe et d'arriver enfin à sortir du silence où ils nous avaient tenues. Dans ce but, la narratrice explore son propre corps. Plonger en soi-même jusque dans son ventre semble la condition pour que la violence (la colère, la rage, l'instinct de survie) retrouve son caractère libérateur et ne soit plus une arme retournée contre soi. Son corps en proie à la démesure, telle une ogresse, elle mange, absorbe, prend tout en elle, digère, transforme. L'univers revu et corrigé passe par son ventre:

Je dis je suis une effroyable déesse; je mange vos langues vos têtes vos cœurs, ceuxlà qui ne savent pas aimer. J'absorbe l'économie du savoir et l'écoule aux marchés de la déroute. Dans ma panse d'obscurité, je digère les doutes, les hésitations. (13);

au plus sombre je me renouvelle. J'appuie ma fatigue sur l'ombre comme s'il s'agissait d'une embrasure. L'espace, la durée, la matière oxydent les humiliations, façonnent le pain gris, labourent les bassesses. (66)

C'est sans doute dans cette volonté de considérer la violence, cette pulsion inhérente à l'humain, comme une force de vie, que les trois auteurs se rapprochent le plus. Or pour cela, il faut activer la mémoire, remonter jusqu'aux blessures de l'enfance et bien au-delà :

Je dis que nous errons au travers les années, la tête enfouie, coupée, disloquée dans le ventre.

Après que la pluie eut puisé l'aube et déshabillé les mères, des poussières de verbes contractés parmi les fougères cherchent à renverser le silence. (20)

Dans ce poème, la confusion temporelle incite à penser la préhistoire et l'histoire ensemble, à ne plus voir de clivage entre les générations, les époques. C'est sur la multiplication des temporalités et des espaces que mise l'auteure, car dans ce choc des réalités, comme le figure la forme des textes (courts assemblages de phrases sans liens appuyés, mais dont la juxtaposition fait sens et image - parfois de manière fulgurante), les conditions semblent réunies pour que les «gisantes», une fois «lavée[s] de la honte», se révèlent «météores» (11).

Ainsi, par un bel anachronisme, la narratrice se retrouve en présence de Josée Yvon. À la faveur de cette rencontre et de la sensualité complice qui se développe entre elles (le désir est plus fort que la honte), l'enfance sera démuselée, les squelettes du passé libérés, le nœud identitaire dénoué. Dans le rapprochement des corps, l'abandon aux caresses, la femme se dévoile dans toute sa complexité:

C'est la fête, nous buvons des incendies de femmes qui s'étirent sexe dehors langue râpeuse pas sages pas propres pas habillées pour sortir. L'éblouissement met la tendresse en exil, me révèle Josée. Nous faisons tout n'importe comment, même l'amour, accouplées à la rage. (36; l'auteure souligne.) 
Cette rencontre l'incite à défier la peur, la difficulté d'aimer, et à briser le cercle vicieux dans lequel les femmes se trouvent prises depuis la nuit des temps ${ }^{12}$.

Dans la dernière partie, la voix s'affiche plurielle; la femme, résolument sortie du silence, affranchie de la honte, intègre toutes les identités, et la contradiction cède à la fluidité:

Ici m'arrive ce que l'on peut attendre. Ici change tout le temps. Ici la blessure en même temps que la joie. Ici simplement la blessure. Ici simplement la joie. Ici ta présence et ma voix. Ici une table, une étoile, la galaxie. (63)

Ici parle la Méduse, comme l'indique le titre de la suite ("Comme si j’étais la Méduse»), une créature mythique complexe, ambiguë, à la fois victime et menace ${ }^{13}$. Parler comme la Méduse, c'est en même temps renoncer au statut de géante et reconnaître sa force, affronter la mort qui vous guette depuis l'enfance sans laisser ses yeux vous pétrifier. C'est prendre le parti de la multiplicité en logeant en soi l'espace ainsi déplié. Comme le déterminant précédant le nom invite à le lire, c'est aussi, par le recours à l'éclatement temporel, permettre à son corps de redevenir ductile, souple comme celui de la créature marine, au point de prendre toutes les formes. Écrire «ici», c'est faire acte de présence comme on fait acte de foi, se dire que toute violence est un amour blessé, une vie empêchée, et qu'en tout poème gît une prière, peutêtre, qu'une fois retourné contre soi il adresse à la vie, celle qui comme aux chats nous arrache des cris, mais à laquelle, parfois, l'écriture nous rallie, pour en desceller la beauté. Et qui sait, peut-être en tient-il aussi à nous, lecteurs de poèmes, d'ensemencer la terre que les mots labourent afin de permettre des récoltes nouvelles.

12 «Je n'échappe à rien. Je bois une eau qui ne m’hydrate plus. J'escalade le mur que je porte en moi. Sur le plancher de la cuisine, une famille gît dans sa mare. Je suis en fuite. Je n'échappe à rien. » (39)

13 Selon une version du mythe grec, Méduse était à l'origine une belle jeune fille que Poséidon, épris d'elle, aurait violée dans un temple d'Athéna, laquelle se serait vengée sur la victime en la transformant en Gorgone dont les yeux ont le pouvoir de pétrifier quiconque la regarde. Persée, après l'avoir décapitée, remettra son masque à Athéna, qui le fixera à son bouclier en symbole de puissance. 